\title{
Antimicrobial Peptide Human Neutrophil Peptide 1 as a Potential Link Between Chronic Inflammation and Ductal Adenocarcinoma of the Pancreas
}

\author{
Thomas Pausch, MD, * Sarah Adolph, * Klaus Felix, PhD, * Andrea S. Bauer, PhD, $\dagger$ \\ Frank Bergmann, MD, $\neq$ Jens Werner, $M D$, * $\xi$ and Werner Hartwig, $M D^{*} / /$
}

\begin{abstract}
Objectives: Defensins are antimicrobial peptides playing a role in innate immunity, in epithelial cell regeneration, and in carcinogenesis of inflammation-triggered malignancies. We analyzed this role in pancreatic ductal adenocarcinoma (PDAC) in the context of its association with chronic pancreatitis (CP).

Methods: Human tissue of healthy pancreas, CP, and PDAC was screened for defensins by immunohistochemistry. Defensin $\alpha 1$ (human neutrophil peptide 1 [HNP-1]) expression was validated using mass spectrometry and microarray analysis. Human neutrophil peptide 1 expression and influences of proinflammatory cytokines (tumor necrosis factor $\alpha$, interleukin $1 \beta$, and interferon $\gamma$ ) were studied in human pancreatic cancer cells (Colo 357, T3M4, PANC-1) and normal human pancreatic duct epithelial cells (HPDE). Results: Accumulation of HNP-1 in malignant pancreatic ductal epithelia was seen. Spectrometry showed increased expression of HNP-1 in CP and even more in PDAC. At RNA level, no significant regulation was found. In cancer cells, HNP-1 expression was significantly higher than in HPDE. Proinflammatory cytokines significantly led to increased HNP-1 levels in culture supernatants and decreased levels in lysates of cancer cells. In HPDE cytokines significantly decreased HNP-1 levels.
\end{abstract}

Conclusions: Inflammatory regulation of HNP-1 in PDAC tissue and cells indicates that HNP-1 may be a link between chronic inflammation and malignant transformation in the pancreas.

Key Words: antimicrobial peptides, pancreas, adenocarcinoma, chronic pancreatitis

(Pancreas 2018;47: 561-567)

P ancreatic ductal adenocarcinoma (PDAC) is one of the most deadly tumors with less than $5 \%$ survival after 5 years; it is among the 5 leading causes of tumor death in the United States. ${ }^{1}$ Early surgery remains the only potentially curative treatment option, but early tumor detection is difficult and the cancer

From the *Department of Surgery General, Visceral and Transplantation Surgery, University of Heidelberg; †Division of Functional Genome Analysis, German Cancer Research Center; IInstitute of Pathology, University of Heidelberg, Heidelberg; §Department of General, Visceral, Vascular and Transplantation Surgery, University of Munich, München; and ||Department of General, Visceral and Vascular Surgery, Evangelisches Krankenhaus Düsseldorf, Düsseldorf, Germany.

Received for publication July 10, 2017; accepted March 2, 2018.

Address correspondence to: Thomas Pausch, MD, Department of General,

Visceral and Transplantation Surgery, University of Heidelberg, Im

Neuenheimer Feld 110, 69120 Heidelberg, Germany

(e-mail: thomas.pausch@med.uni-heidelberg.de).

The authors declare no conflict of interest.

Presented at the 44th Annual Meeting of the American Pancreatic Association,

October 30 to November 2, 2013, Miami, FL, and at the 44th Meeting of the

European Pancreatic Club, June 20-23, 2012, Prague, Czech Republic.

Supplemental digital contents are available for this article. Direct URL citations appear in the printed text and are provided in the HTML and PDF versions of this article on the journal's Web site (www.pancreasjournal.com).

Copyright (C) 2018 Wolters Kluwer Health, Inc. All rights reserved.

DOI: 10.1097/MPA.0000000000001054 still largely defies any medical therapy. ${ }^{2}$ Recent progress has been made in the understanding of carcinogenesis with advances in molecular biology, but until now, only a little therapeutic progress has resulted. Epigenetic changes and familial syndromes have been associated with PDAC, ${ }^{3,4}$ which still does not explain the disease epidemiologically. ${ }^{5}$ The most consistent epidemiological risk factor for developing PDAC is chronic pancreatitis (CP). ${ }^{6,7}$ There is an increasingly high risk of pancreatic cancer in individuals with hereditary pancreatitis over the age of 50 years $^{8}$ with a cumulative lifetime risk of $40 \%$ at the age of 70 years. ${ }^{9}$ Nevertheless, the genetic changes that cause $\mathrm{CP}$ have not been found in sporadic pancreatic cancer. ${ }^{10}$ Consequently, it has been postulated that genetic susceptibility in interaction with environmental factors and an altered immune response causes recurrent and largely asymptomatic pancreatic injury, which progresses to chronic inflammation, fibrosis, and over time to malignant transformation of the pancreas. ${ }^{10,11}$

The concept of cancer diseases as "wounds that do not heal" goes back to Virchow's observation of leukocytes in malignant tissue in $1863 .^{12}$ The difference between wound healing and cancer is the lack of self-limitation of the proliferation of the malignant cells. During carcinogenesis, the mechanisms that would physiologically repair or eliminate the dysplastic cells fail. Tumor cells in cooperation with their stroma abuse the immune system to recruit or deceive various inflammatory cells and create an enriched cytokine milieu, which protects the tumor cells and supports their growth. ${ }^{13}$ So-called defensins are important immune factors. These small cationic antimicrobial proteins link the innate to the adaptive immune system. Defensins are expressed by various epithelia and leucocytes (some of them are named human neutrophil peptides [HNPs]), to protect the body's surfaces against pathogens. ${ }^{14,15}$ During the recurrent asymptomatic inflammatory processes that lead to carcinogenesis in the pancreas, these immune factors may possibly be switched-off or abused by cancer cells. Pancreatic ductal adenocarcinoma is known for its strong desmoplastic reaction, which involves complex intercellular interactions in the tumor microenvironment that promote malignant transformation, invasion, metastasis, and resistance to chemotherapy. ${ }^{16,17}$ Furthermore, defensins in general are well known for participating in multiple regulations of acute and chronic inflammatory, regenerative, and proliferative processes. ${ }^{15,18}$

Defensins have multiple and partly contrary effects on cancer cells. Some defensins disturb the development of malignant cells $^{19,20}$ or destroy them directly ${ }^{15,21}$ or via immunological modulation. ${ }^{22}$ In contrast, other defensins support the proliferation or migration of malignant cells. ${ }^{20,23}$ The differential expression of defensins has been described in several gastrointestinal tumors. ${ }^{24,25}$ In the pathogenesis of CP and PDAC, defensins seem to play a certain role. Although their pathogenetic role is not yet fully clarified, defensins have been detected in pancreatic tissue, in fetal and adult acinar cells, and also in pancreatic juice. ${ }^{26,27}$ Likewise, severe acute pancreatitis has been associated with polymorphisms 
of defensin genes, ${ }^{28}$ and defensins have been detected in pancreatic metaplasia. $^{29}$

We assume that disturbances of the regulative function of defensins, possibly triggered by chronic inflammation, could promote malignant transformation and tumor growth within the pancreas. During the inflammatory process, defensins may either be switched off or the cancer may abuse their functions. The aim of the present study was to analyze the role of defensins in the carcinogenesis of PDAC and its association with $\mathrm{CP}$.

\section{MATERIALS AND METHODS}

\section{Patients and Specimens}

Tissue samples were obtained from patients in whom pancreatic resection had been performed for PDAC and CP at the Department of General Surgery, University Hospital in Heidelberg, Germany between 2001 and 2010. Patients had given informed consent to the collection of tissue. Normal pancreas specimens were collected from patients who had undergone resection of pancreatic tissue with nonmalignant and noninflammatory diagnoses (eg. benign cystic lesions). This collection was performed in accordance to the Helsinki Declaration and approved by the Ethics Committee of the University of Heidelberg (Votum 301/2001). A pathologist ascertained that the diagnosis corresponded to the World Health Organization classification. All specimens for use in immunhistochemistry were fixed in paraformaldehyde solution for 12 to 24 hours immediately after surgery and were paraffin embedded for histological analysis. Specimens for use in expression analysis and genome analysis were immediately snap frozen in liquid nitrogen and stored at $-80^{\circ} \mathrm{C}$ until use.

\section{Immunhistochemistry in Pancreatic Tissue}

Human tissue samples of the normal pancreas, CP, and PDAC were screened for defensins using immunohistochemistry ( $n=20$ per group). Paraffin-embedded sections $(4-\mu \mathrm{m})$ of specimens of normal pancreas, CP, and PDAC were deparaffinized (Roticlear, Carl Roth, Karlsruhe, Germany), rehydrated in progressively decreasing concentrations of ethanol, and put on slides. For antigen retrieval, the slides were pretreated in citrate buffer $(\mathrm{pH}$ 6.0) for $2 \times 7$ minutes in a microwave oven. They were washed with water before they were incubated in $3 \% \mathrm{H}_{2} \mathrm{O}_{2} /$ methanol at room temperature for 10 minutes to quench endogenous peroxidase activity. Slides were rinsed in Tris-buffered saline (TBS, pH 7.4; Sigma-Aldrich, St Louis, Mo) with $1 \%$ bovine serum albumin (BSA; Sigma-Aldrich) for $2 \times 10$ minutes to block nonspecific activity. Incubation of the slides with the following corresponding primary antibodies was performed overnight in a $4^{\circ} \mathrm{C}$ humidity chamber (data are presented in Table 1): antihuman defensin $\beta 1, \beta 2, \beta 3, \alpha 1, \alpha 3, \alpha 5$, and $\alpha 6$. For the staining of the infiltrating inflammatory cells, anti-human CD15 primary antibody was used according to the manufacturer's instructions (Table 1). Again, the slides were washed with TBS/BSA and $0.05 \%$ Tween (Sigma-Aldrich) for $3 \times 10$ minutes and with TBS/BSA for $2 \times 10$ minutes. Appropriate horseradish peroxidaselinked goat anti-rabbit (K4003, DAKO, Glostrup, Denmark) or goat anti-mouse (K4001, DAKO) secondary antibodies were added for incubation in a humidity chamber at room temperature for 90 minutes, followed by reaction with diaminobenzidine and counterstaining with Mayer's hematoxylin (S3309, DAKO) before the mounting of the specimens. Corresponding positive control sections of human tissue from skeletal muscle or tonsil were incubated with the primary antibodies to ensure adequate antibody functioning. Negative controls were incubated in the absence of the primary antibodies. A pancreas-specialized pathologist (F.B.) evaluated the immunohistological staining.

\section{Expression Analysis of HNP-1 in Pancreatic Tissue}

The protein lysates from human pancreatic tissue of normal pancreas, $\mathrm{CP}$, and PDAC ( $\mathrm{n}=10$ per group), which were selected by evaluation of representative HE stainings from each tissue sample by a pathologist (F.B.), were profiled by applying mass spectrometry (surface-enhanced laser desorbtion/ionization time-offlight mass spectrometry [SELDI-TOF-MS]) and by the use of a ProteinChip Reader (PBS II; Ciphergen Biosystems, Fremont, Calif). Pancreatic tissue was broken down in radioimmunoprecipitation assay buffer (RIPA buffer, Sigma-Aldrich) to lysate by repeated aspiration through a cannula before ultrasound-homogenization. BCA Protein Assay Kit (Thermo Scientific, Waltham, Mass) was used according to the manufacturer's protocol to equalize the total amount of proteins between the probes. All probes were immediately frozen at $-80^{\circ} \mathrm{C}$ and stored until analysis.

Probes were put on PS20 ProteinChip preactivated surface arrays (Ciphergen Biosystems) for analysis as previously described. ${ }^{30}$ The chips were loaded with 600 ng protein G (P4689, SigmaAldrich) per spot and preincubated in a humidity chamber at room temperature for 3 hours. After inactivation with Tris-buffer $(\mathrm{pH} 8)$, they were incubated for another 30 minutes followed by washing another 2 times with phosphate buffered saline (PBS, SigmaAldrich) 1\% Triton X-100 (Sigma-Aldrich) and once with PBS only. Spots were then loaded with $500 \mathrm{ng}$ anti- $\alpha 1$ defensin antibody each (Table 1). Again, the chips were incubated in a humidity chamber at room temperature for 3 hours. After another washing with Tris-buffer, the spots were washed 3 times with a buffer containing $100 \mathrm{mmol} / \mathrm{L}$ sodium phosphate (pH 7.4), $500 \mathrm{mmol} / \mathrm{L}$ sodium chloride, and $0.05 \%$ Triton $\mathrm{X}-100$. Tissue protein lysates $(120 \mu \mathrm{L})$ were applied to the spots using a ProteinChip cassette-compatible bioprocessor (Bio-Rad, Hercules, Calif), and the chips were incubated in a humidity chamber at $4^{\circ} \mathrm{C}$ overnight. Subsequently, the chips were washed 3 times with a sodium phosphate/sodium chloride/Triton X-100 buffer and 2 times with water before $1 \mu \mathrm{L}$ of ProteinChip sinapinic acid energy-absorbing molecules (Bio-Rad) was added to each spot. The spots were air-dried, and the last step was repeated. The ion mass of the proteins was analyzed on arrays using the ProteinChip Reader. Mass spectra of the analyzed probes were normalized with total ion current and signal cluster analysis of signals exhibiting a signal-to-noise ratio of at least 2 .

\section{Pancreatic Tissue Genome Analysis}

Human pancreatic tissue samples were collected during surgery and were snap frozen in liquid nitrogen directly after resection and subsequently stored at $-80^{\circ} \mathrm{C}$ until being used in the analysis as described above. The frozen tissue samples were cut into slices of 10 to $20 \mu \mathrm{m}$ thickness with a cryotome, submerged in liquid nitrogen, and gently ground by 3 turns of a polypropylene micropestle (Eppendorf, Hamburg, Germany) that fits into 2-mL Eppendorf tubes.

Total RNA from individual tissue samples $(n=195$ of PDAC, $n=59$ of CP, $n=41$ of healthy pancreatic tissues) was isolated with the AllPrep Isolation kit (Qiagen, Hilden, Germany), following the protocol suggested by the manufacturer, and analyzed on Sentrix Human-6v3 Whole Genome Expression BeadChips (Sentrix Human WG-6; Illumina, San Diego, Calif). To synthesize the first and second strand cDNA and amplify biotinylated cRNA from the total RNA, an Illumina Totalprep RNA Amplification Kit (Illumina) was used. The hybridization to the BeadChip was performed. All this was done according to the manufacturer's instructions. A maximum of $10 \mu \mathrm{L}$ cRNA was mixed with a $20 \mu \mathrm{L}$ 
TABLE 1. Primary Antibodies Used in Immunohistochemical Stainings and SELDI-TOF-MS

\begin{tabular}{|c|c|c|c|c|}
\hline Antigen & Antibody & Type/Host & Manufacturer & Dilution Used \\
\hline \multicolumn{5}{|l|}{ Immunohistochemistry } \\
\hline Human defensin $\beta 1$ & HBD12-A & Polyclonal/rabbit & Alpha Diagnostics, San Antonio, Tex & $1: 50$ \\
\hline Human defensin $\beta 2$ & $14338-\mathrm{v}$ & Antiserum/rabbit & Peptide Institute, Osaka, Japan & $1: 200$ \\
\hline Human defensin $\beta 3$ & LS-B86 & Polyclonal/rabbit & Lifespan Biosciences, Seattle, Wash & $1: 100$ \\
\hline Human defensin $\alpha 1$ & BS3505 & Polyclonal/rabbit & Bioworld Technology, Minn & $1: 100$ \\
\hline Human defensin $\alpha 3$ & NBP1-19734 & Polyclonal/rabbit & Novus Biologicals, Littleton, Colo & $1: 100$ \\
\hline Human defensin $\alpha 5$ & NB110-60002 & Monoclonal/mouse & Novus Biologicals, Littleton, Colo & $1: 50$ \\
\hline Human defensin $\alpha 6$ & HPA019462 & Polyclonal/rabbit & Atlas Antibodies, Stockholm, Sweden & $1: 1000$ \\
\hline Human CD15 & M3631 & Monoclonal/mouse & DAKO, Glostrup, Denmark & $1: 50$ \\
\hline \multicolumn{5}{|l|}{ SELDI-TOF-MS } \\
\hline Human defensin $\alpha 1$ & ab90486 & Monoclonal/mouse & Abcam, Cambridge, United Kingdom & - \\
\hline
\end{tabular}

GEX-HYB hybridization solution. The preheated $30 \mu \mathrm{L}$ assay sample was dispensed onto the large sample port of each array and incubated for 18 hours at $58^{\circ} \mathrm{C}$. After hybridization, the samples were washed according to the protocol and scanned with a BeadArray Reader (Illumina).

\section{In Vitro Analysis of Defensin Expression in Pancreatic Cell Cultures}

Three representative human pancreatic cancer cell lines were chosen for experiments (for cell growth curves see Supplemental Digital Content, http://links.lww.com/MPA/A652): the slow-growing cell line Colo 357 originated from a lymphatic metastasis of an adenocarcinoma of the pancreas (provided by R. Metzgar, Duke University, Durham, NC, Px 9-28); the PANC-1 cell line originated from cells of ductal pancreatic adenocarcinoma growing at intermediate speed (ATCC, Rockville, Md, Px 16-27); and the fast-growing cell line T3M4 also originated from a lymphatic metastasis of an exocrine tumor of the pancreas (provided by R. Metzgar, Duke University, Durham, NC, Px 14-31).

Cell lines were grown in complete medium (RPMI 1640, Gibco Invitrogen, Carlsbad, Calif) containing 10\% fetal bovine serum (Pan Biotech, Aidenbach, Germany) and 1\% penicillinstreptomycin (Gibco Invitrogen), and incubated at $37^{\circ} \mathrm{C}$ in a $3.5 \% \mathrm{CO}_{2}$ humidified atmosphere. For passaging, the cells were washed with $10 \mathrm{~mL}$ Dulbecco's PBS (Promocell, Heidelberg, Germany) before detachment by Trypsin $0.25 \%$ ethylenediaminetetraacetic acid (Gibco Invitrogen) was stopped by adding complete Roswell Park Memorial Institute medium (RPMI medium, Sigma-Aldrich).

To compare human neutrophil peptide 1 (HNP-1) expression by malignant and nonmalignant human pancreas cells, probes from immortalized human normal human pancreatic duct epithelial cell line cultures (HPDE; provided by M.S. Tsao, University of Toronto, Toronto, Canada) were generated. HPDE were grown in Keratinocyte-SFM with L-glutamine, without calcium chloride containing bovine pituitary extract ( $25 \mu \mathrm{g} / \mathrm{mL}$, Gibco Invitrogen) and recombinant epithelial grwoth factor $(0.2 \mathrm{ng} / \mathrm{mL}$, Gibco Invitrogen $)$.

All cells were seeded in 6-well plates. After 24 hours of adherence, the medium was changed into a serum-free special medium for immunological studies (AIM V medium, Gibco Invitrogen) to prevent interference of serum proteins with cytokines.

After incubation of cells for 0 hours, 24 hours, and 48 hours, the medium was aspirated and dead cells were discarded after centrifugation for 6 minutes at $1200 \mathrm{rpm}$. Supernatant and cell pellets were separated. Cell pellets were incubated in RIPA buffer on ice for 10 minutes and mechanically resuspended before breakdown to lysate by repeated aspiration through a cannula. Lysate was centrifuged for 15 minutes at $13,000 \mathrm{rpm}$, and cell debris was discarded. All probes ( $n=6-9$ per group) were immediately frozen at $-80^{\circ} \mathrm{C}$ and stored until analysis.

\section{In Vitro Stimulation of Cancer Cells With Proinflammatory Cytokines}

The effects of proinflammatory cytokines on tumor cellspecific expression of HNP-1 were studied by adding recombinant bioactive human tumor necrosis factor $\alpha(\mathrm{TNF}-\alpha)$, recombinant bioactive human interleukin (IL) $1 \beta$, and recombinant bioactive human interferon (IFN) $\gamma$ (all R\&D Systems, Minneapolis, Minn) to the cell cultures. Cytokines were all added at a concentration of $100 \mathrm{ng} / \mathrm{mL}$. The concentration of the cytokines was set at this level after a pilot series of cell growth assays. Increasing concentration levels of cytokines up to $100 \mathrm{ng} / \mathrm{mL}$ of the cytokines were shown to have no influence on the growth rate and survival of the cells (data not shown). Probes of supernatants and lysates $(n=6-9$ per group) were collected at 0 hours, 24 hours, and 48 hours after incubation for analysis by SELDI-TOF-MS, as described above.

\section{Expression Analysis in Cell Culture}

SELDI-TOF-MS analysis of cell lysates and supernatants of human pancreatic cancer cell line cultures and HPDE cultures was performed in a way analogous to the tissue analysis described previously with the application of $200 \mu \mathrm{L}$ of cell-culture supernatants and $75 \mu \mathrm{L}$ of cell lysates per spot.

\section{Data Analysis}

Statistical analyses of defensin protein expression were performed using GraphPad Prism (version 3.0; GraphPad Software, San Diego, Calif). Student's $t$-test (unpaired, two-tailed) was applied to compute $P$ values. For statistical analyses of genomic expression, R (R Foundation, Vienna, Austria, http://www.R-project. org) was used. Significant differentially expressed genes were detected using the limma package of R/Bioconductor by pairwise comparisons of the sample groups. ${ }^{31}$ The resulting $P$ values were adjusted for multiple testing using Benjamini-Hochberg's false discovery rate method. ${ }^{32}$

\section{RESULTS}

Human neutrophil peptide 1 was specifically detected in tissue samples by the immunohistochemical staining. Protein expression was most pronounced in the apical cytoplasm of malignant ductal pancreatic epithelial cells (Fig. 1) but also to a lesser degree and diffusely distributed in CP. In healthy pancreas, HNP-1 was detected 


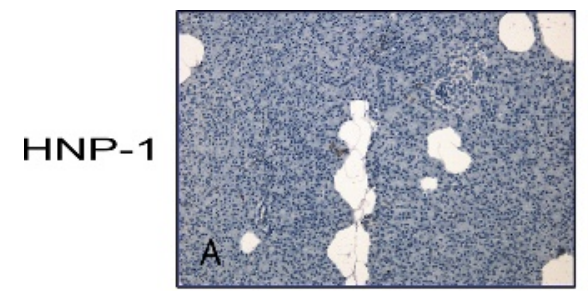

Healthy

Pancreas

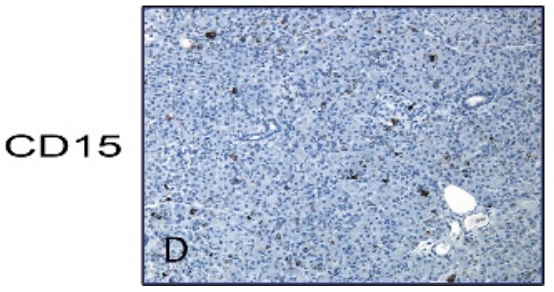

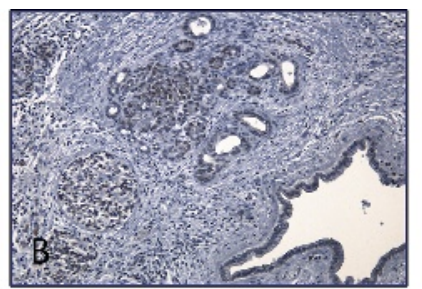

Chronic

Pancreas

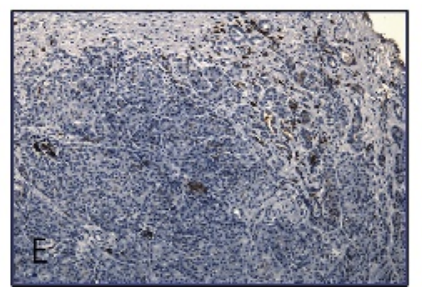

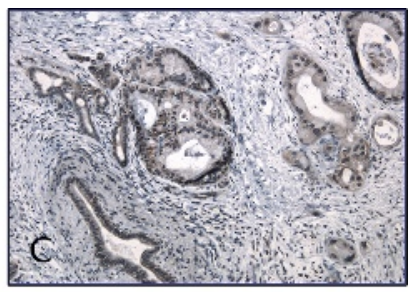

Ductal
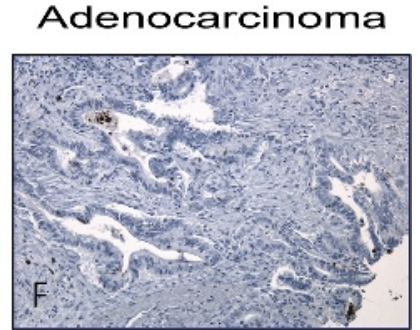

FIGURE 1. Immunohistochemical detection of HNP-1 in human pancreatic tissue. Immunohistochemical staining of HNP-1 shows scarcely detection in healthy pancreatic tissue (A), light diffusely distributed expression in CP (B) and strong expression in PDAC with emphasis on apical cytoplasm of malignant ductal epithelial cells (C). Lower pictures of anti-CD15 staining (D-F) show accumulation of inflammatory cells in CP, not in healthy pancreas and PDAC. Images are representative of 20 independent stainings per group (original magnifications, $\times 20$ ).

Editor's note: A color image accompanies the online version of this article.

only scarcely. Likewise, defensins $\alpha 3$ (HNP-3) and $\beta 5$ human beta defensin (HBD-5) were stained in pancreatic duct epithelia but without obvious differences between healthy pancreas, $\mathrm{CP}$, and PDAC (not shown). Defensin $\beta 1$ (HBD-1) was stained in healthy pancreas, but it could hardly be found in CP and PDAC (not shown). Immunohistochemical staining against human CD15 showed the accumulation of inflammatory cells in the tissue of $\mathrm{CP}$, but not in healthy pancreas and PDAC (Fig. 1).

In the mass spectrometric analysis, slightly elevated expression levels of HNP-1 were found in CP compared with healthy pancreas $(P=0.235)$. Expression levels were significantly increased in PDAC compared with the healthy pancreas $(P=0.003)$ and to $\mathrm{CP}$ $(P=0.024)$ (Fig. 2).

A typical finding of SELDI-TOF-MS analysis is demonstrated in Figure 3. The peak of the ionized HNP-1 protein is observed at $3.45 \mathrm{kDa}$.

On a nuclear level, HNP-1 was represented by 2 different probe sequences (ILMN_1679357 and ILMN_2193213) on Illumina Whole Genome Expression Bead Chips with average q-normalized log expression level of 6.17 and 5.97. No significant differences were found between healthy pancreas, $\mathrm{CP}$, and PDAC in the expression of HNP-1 on RNA level (healthy pancreas vs CP $P=0.586$ and 0.914 ; healthy pancreas vs PDAC $P=0.226$ and 0.977; CP vs PDAC $P=0.701$ and 0.751 ).

In vitro experiments verified the expression of HNP-1 in all of the 3 pancreatic cancer cell lines with higher expression levels in cell lysates compared with supernatants (mean, $71 \mathrm{ng} / \mathrm{mL}$ total protein concentration in lysates and $17 \mathrm{ng} / \mathrm{mL}$ in supernatants). Smaller amounts of HNP-1 were found in nonmalignant pancreatic ductal epithelial cells (mean, $34 \mathrm{ng} / \mathrm{mg}$ total protein concentration in lysates and $5 \mathrm{ng} / \mathrm{mg}$ in supernatants). However, HNP-1 expression was significantly higher in supernatants $(P=0.0004)$ and also in lysates $(P=0.04)$ of pancreatic cancer cell lines (Fig. 4) compared with nonmalignant pancreatic ductal epithelial cells at 0 to 48 hours of incubation.

Cancer cells that had been stimulated in vitro with proinflammatory cytokines demonstrated significantly increased expression levels of HNP-1 in cell culture supernatants. When Colo 357 cells were stimulated with IL-1 $\beta$, significantly higher levels of HNP-1 were measured in the supernatants compared with unstimulated cells at 48 hours $(P=0.008)$ (Fig. 5A). Likewise, after stimulation of PANC-1 and T3M4 cells with IL-1 $\beta$ (Figs. 5B, D) or IFN- $\gamma$ (Figs. 5C, E), HNP-1 protein levels in the supernatants were significantly higher than in the unstimulated situation at 48 hours (PANC-1, $P=0.0013$ and $P=0.007$; T3M4, $P=0.003$ and $P=0.027$, respectively).

Cell lysates were found to contain only temporarily decreased levels of HNP-1 after inflammatory stimulation. At 48 hours, a significant decrease in the amount of HNP-1 in stimulated cell lysates was only seen if all cancer cell lines were summarized before calculation $(P<0.001$, data not shown). If cell lines were analyzed separately, significant decreases were only found in lysates of T3M4 cells at 24 hours after incubation with TNF- $\alpha$, and IL- $1 \beta$ at 48 hours after incubation with TNF- $\alpha(P=0.026, P=0.0002$, and $P=0.009$, data not shown).

When nonmalignant HPDE cells had been stimulated with TNF- $\alpha$, IL-1 $\beta$, or IFN- $\gamma$, no increases of HNP-1 were seen at all. A significant decrease of HNP-1 expression was found in supernatants and in lysates of HPDE cells at 48 hours after stimulation

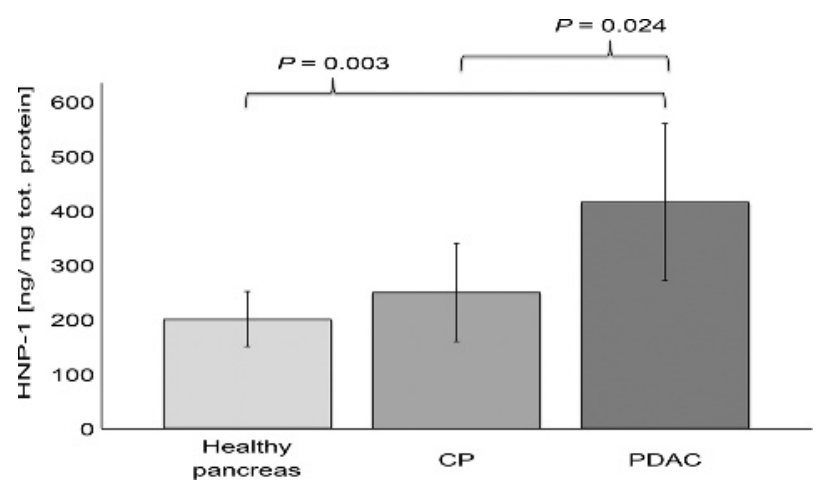

FIGURE 2. Human neutrophil peptide 1 expression in pancreatic tissue. In mass spectrometric analysis, elevated expression levels of HNP-1 were found in CP compared with healthy pancreas (statistically not significant, $P=0.235$ ). In addition, expression levels were significantly increased in PDAC compared with healthy pancreas and compared with CP ( $n=7$ per group). Tot. indicates total. 


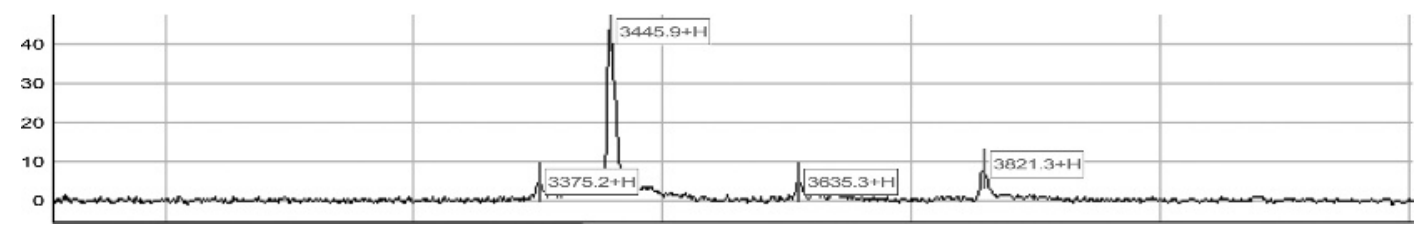

FIGURE 3. Example peak of HNP-1 in SELDI-TOF-MS analysis (3.45 kDa).

with TNF- $\alpha$, IL-1 $\beta$, and IFN- $\gamma$ (for all supernatants $P=0.004$; for all lysates $P=0.006$, data not shown).

\section{DISCUSSION}

To the best of our knowledge, the present study shows for the first time that an antimicrobial peptide, in particular HNP-1, is strongly expressed in malignant human pancreatic tissue in vivo and in malignant human pancreatic epithelial cells in vitro. Proinflammatory stimuli induced the secretion of HNP-1 by pancreatic tumor cells and may therefore indicate a possible role of defensins in inflammation-related pancreatic carcinogenesis.

Defensins are physiologically expressed by human epithelia and immune cells to protect our body against various pathogens. Being part of both the innate and adaptive immune systems, they link the first to the second line of defense in the battles on our body's surfaces. ${ }^{15}$ Being important modulators of several epithelial intracellular mechanisms, they act as mediators of intercellular interactions between multiple cells in the epithelial surroundings. Thereby, defensins have an effect on physiological regenerative and proliferative processes ${ }^{18}$ and they participate in the regulation of acute and chronic inflammatory processes. ${ }^{15}$ Unsurprisingly, their role has been studied in conditions of uncontrolled proliferation, and they have been described as having multiple influences on cancer cells; for example, HNP-1 to -3 have been demonstrated to promote proliferation and invasiveness of renal, bladder, oral squamous cell carcinoma, and invasive head and neck carcinoma, and they have been found to be upregulated in these cancers. ${ }^{20,23,33,34}$ Defensins also seem to play an important role in the carcinogenesis of gastrointestinal tumors. Human neutrophil peptide 1 expression in the tissue of intestinal-type gastric cancer has been correlated with reduced survival, ${ }^{24}$ and the overexpression of HNP-1 to -3 has been shown in tumor cells, serum, and stool of patients with colorectal cancer. ${ }^{25}$ Alterations of defensin expression during malignant transformation caused by permanent proinflammatory stimulation have been described, for example, in colorectal cancer consecutive to inflammatory bowel disease. ${ }^{35}$ Acute and chronic pancreatitis on the other hand may occur as an extra-intestinal comorbidity of inflammatory bowel disease. ${ }^{36}$ Various defensins have been postulated to play a role in pancreatic diseases. Human beta defensin 1 (HBD-1) and HNP-3 secreted by acinar cells may prevent the invasion of bacteria from the proximal small intestine. ${ }^{26,27}$ Polymorphisms of HBD-1 and HBD-2 have been associated with severe acute pancreatitis, ${ }^{28}$ and the detection of human defensin $\alpha 5$ (HAD-5) in pancreatic metaplasia has been seen as an indicator of the adenoma-carcinoma sequence. ${ }^{29}$

In the present study, HNP-1 was found to be expressed at a significantly higher level in malignant human pancreatic tissue than in healthy pancreas or in CP. These findings support the hypothesis that defensins, in particular HNP-1, may represent an important link between chronic inflammation and malignant transformation in the pancreas. Because physiologically HNP-1 is mainly expressed by granulocytes, it can be hypothesized that the concentrations of protein measured in the present study could be biased by the presence of inflammatory cells in the tumor microenvironment. Three findings contradict this hypothesis: first, immunohistochemical screening for defensins showed specific HNP-1 staining of pancreatic ductal epithelial cells; second, staining of the neutrophil marker CD15 showed the specific accumulation of inflammatory cells in our CP tissue samples and not in our PDAC tissue samples, contrary to the HNP-1 staining; and third, quantitative analysis of HNP-1 expression was performed subsequent to the careful selection of representative samples by a specialized pathologist and quantification showed significantly higher amounts of HNP-1 expressed in PDAC tissue compared with CP tissue. Therefore, the fact that HNP-1 was highly expressed in malignant pancreatic tissue and by proinflammatory-stimulated pancreatic cancer cells but not by inflammatory cells as usual emphasizes the importance of the role HNP-1 might be playing in the inflammatory-triggered development of pancreatic cancer.

Like in human tissue, HNP-1 was strongly expressed by various pancreatic cancer cell lines, but barely by benign pancreatic ductal cells. This HNP-1 overexpression in malignant cells could additionally be triggered by proinflammatory cytokines. Cytokines used for this stimulation are known to be involved in systemic inflammation and in CP. ${ }^{13,37}$ In addition, they are known to increase survival of pancreatic cancer cells via the nuclear

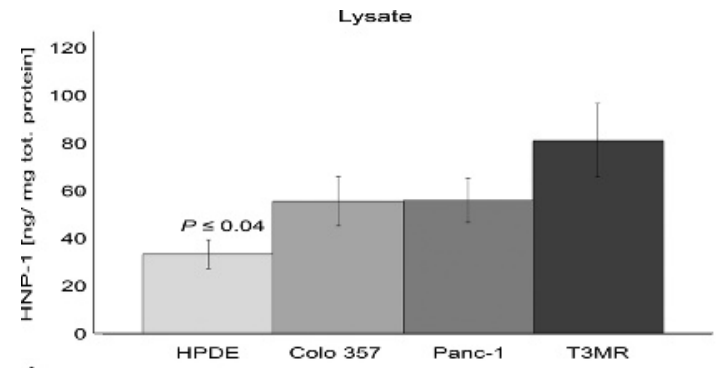

A

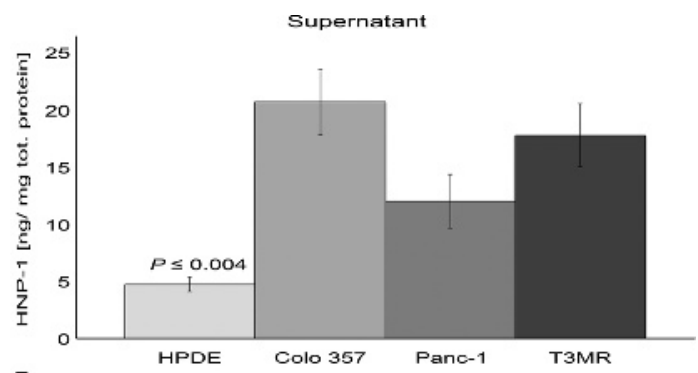

B

FIGURE 4. In vitro analysis of HNP-1 expression in pancreatic adenoma and carcinoma cells. Expression of HNP-1 by pancreatic cancer cell lines was verified in vitro using mass spectrometry. Human neutrophil peptide 1 expression was significantly higher in lysates $(P \leq 0.04)$ (A) and also in supernatants $(P \leq 0.0004)$ (B) of all 3 pancreatic cancer cell lines (T3M4, PANC-1, Colo 357) as compared with benign pancreatic ductal cells (HPDE) (0-48 hours) $(n=18-26$ per group). 


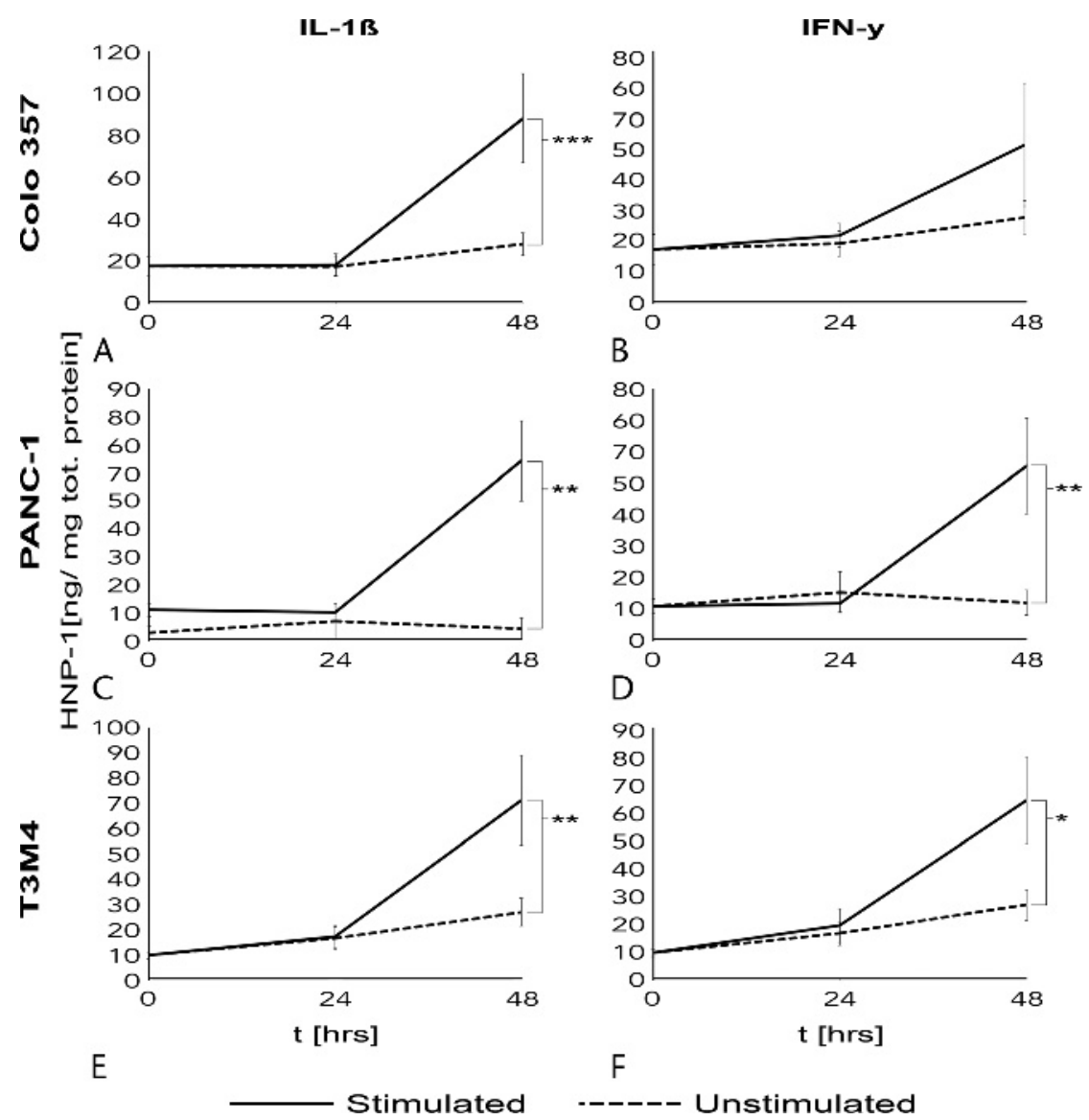

FIGURE 5. In vitro stimulation of pancreatic cancer cell lines (A-B: Colo 357; C-D: PANC-1; E-F: T3M4) with proinflammatory cytokines. Stimulation of all cancer cell lines with IL-1 $\beta(A, C, E)$ and IFN-Y (B, D, F) significantly increased the amount of HNP-1 in the supernatants $(* P<0.05 ; * * P<0.01 ; * * * P<0.001)$.

factor kappa-light-chain-enhancer of activated B-cells and c-Jun $\mathrm{N}$-terminal kinases pathways. ${ }^{38}$ As $\alpha$ defensins are alternatively termed human neutrophil peptides, HNP-1 is highly expressed by granulocytes and secreted from their azurophil granules on infectious and proinflammatory stimulation. ${ }^{15}$ Accordingly, HAD-5 and HAD- 6 are shed by Paneth and intermediate cells in the small intestine. ${ }^{39}$ Thus, we assume that pancreatic cancer cells might secrete HNP-1 by analogous mechanisms. Significant HNP-1 excretion was reflected by a significant increase of HNP-1 concentration in supernatants 48 hours after incubation of cancer cells with proinflammatory cytokines. However, a representative decrease of HNP-1 in stimulated cell lysates was only seen to be statistically significant after 48 hours when all 3 cancer cell lines were summarized for calculation. In separate analysis, a significant decrease of HNP-1 concentration could only be seen in lysates of T3M4. Worth mentioning in this context is the fact that most characteristically the proinflammatory stimulated T3M4 cell is the fastest growing of all 3 pancreatic cancer cell lines used in this study.

Our findings do not claim to describe a singular 1-way pathway from chronic inflammation to cancer via cytokines and defensins. In fact, they solely demonstrate that certain proinflammatory cytokines, which are known to be present in tissue of $\mathrm{CP}^{15}$ can induce HNP1 -expression by pancreatic cancer cells as one potential link between $\mathrm{CP}$ and PDAC.

To discuss the precise nature of this link, one has to consider the 2 domains in which defensins act: on the one hand, they are antimicrobial peptides bringing death to invading bacteria and viruses; on the other hand, they are known to be important intercellular mediators, not only linking innate to adaptive immunity but also regulating multiple regenerative and proliferative processes both physiologically and pathologically in our body. The involvement of microorganisms in carcinogenesis in the pancreas has been studied, and viral infections with mumps and hepatitis B virus as well as bacterial infection with Helicobacter pylori have been described as risk factors. ${ }^{40}$ Specific publications about the pancreas are inconsistent, and the organ itself is not in intense microbiological contact. Therefore, it remains a daring hypothesis to argue that dysbiosis in the pancreas causes inflammatory-triggered malignant transformation with the contribution of antimicrobial factors. It is noteworthy that a strong desmoplastic reaction is a characteristic feature of PDAC, involving multiple cells in the tumor microenvironment and promoting the progression of the disease. ${ }^{16,17,41}$ In this context, the role of defensins as an important link between chronic inflammation and carcinogenesis in the pancreas needs further evaluation. Pancreatic stellate cells (PSCs) appear to be key players in the stromal-epithelial interaction in PDAC, ${ }^{17}$ and their physiologically quiescent status can be ended pathologically by growth factors, cytokines, and oxidative stress. Pancreatic stellate cells are an obvious area of interest concerning the influence of defensins in the pancreas, because PSCs are located basolaterally of pancreatic acinar cells, which again are known to produce HBD- ${ }^{27}$; furthermore, the activation of PSCs has been shown to be caused by alcohol and by bacterial endotoxin lipopolysaccharide. ${ }^{42}$ Upcoming studies about defensins as a potential link 
between $\mathrm{CP}$ and PDAC need to focus on the possible influence of defensins on cell interactions as well as on their possible direct nuclear effect on cancer cells.

\section{ACKNOWLEDGMENT}

The authors thank Prof M.W. Büchler, professor and chairman at the Department of Surgery, University of Heidelberg for his support. Furthermore, they thank Dr N. Giese, K. Schneider, K. Ruf, E. Soyka, and S. Wendler at the European Pancreas Center and $\operatorname{Dr}$ S. Barghorn at AbbVie Germany for technical contributions. Last but not the least, they thank Samuel Waldron for support with figure and text editing.

\section{REFERENCES}

1. Siegel RL, Miller KD, Jemal A. Cancer Statistics, 2017. CA Cancer J Clin. 2017;67:7-30.

2. Hartwig W, Werner J, Jager D, et al. Improvement of surgical results for pancreatic cancer. Lancet Oncol. 2013;14:e476-e485.

3. di Magliano MP, Logsdon CD. Roles for KRAS in pancreatic tumor development and progression. Gastroenterology. 2013;144:1220-1229.

4. Hruban RH, Iacobuzio-Donahue C, Wilentz RE, et al. Molecular pathology of pancreatic cancer. Cancer J. 2001;7:251-258.

5. Hahn SA, Greenhalf B, Ellis I, et al. BRCA2 germline mutations in familial pancreatic carcinoma. J Natl Cancer Inst. 2003;95:214-221.

6. Lowenfels AB, Maisonneuve P, Cavallini G, et al. Pancreatitis and the risk of pancreatic cancer. International Pancreatitis Study Group. NEngl J Med. 1993;328:1433-1437.

7. Malka D, Hammel P, Maire F, et al. Risk of pancreatic adenocarcinoma in chronic pancreatitis. Gut. 2002;51:849-852.

8. Howes N, Lerch MM, Greenhalf W, et al. Clinical and genetic characteristics of hereditary pancreatitis in Europe. Clin Gastroenterol Hepatol. 2004;2:252-261.

9. Vitone LJ, Greenhalf W, Howes NR, et al. Hereditary pancreatitis and secondary screening for early pancreatic cancer. Rocz Akad Med Bialymst. 2005;50:73-84.

10. Whitcomb DC. Inflammation and cancer V. Chronic pancreatitis and pancreatic cancer. Am J Physiol Gastrointest Liver Physiol. 2004;287: G315-G319.

11. Guerra C, Collado M, Navas C, et al. Pancreatitis-induced inflammation contributes to pancreatic cancer by inhibiting oncogene-induced senescence. Cancer Cell. 2011;19:728-739.

12. Balkwill F, Mantovani A. Inflammation and cancer: back to Virchow? Lancet. 2001;357:539-545.

13. Momi N, Kaur S, Krishn SR, et al. Discovering the route from inflammation to pancreatic cancer. Minerva Gastroenterol Dietol. 2012;58: 283-297.

14. Lehrer RI. Multispecific myeloid defensins. Curr Opin Hematol. 2007;14: 16-21.

15. Selsted ME, Ouellette AJ. Mammalian defensins in the antimicrobial immune response. Nat Immunol. 2005;6:551-557.

16. Kikuta K, Masamune A, Watanabe T, et al. Pancreatic stellate cells promote epithelial-mesenchymal transition in pancreatic cancer cells. Biochem Biophys Res Commun. 2010;403:380-384.

17. Omary MB, Lugea A, Lowe AW, et al. The pancreatic stellate cell: a star on the rise in pancreatic diseases. J Clin Invest. 2007;117: 50-59.

18. Droin N, Hendra JB, Ducoroy P, et al. Human defensins as cancer biomarkers and antitumour molecules. J Proteomics. 2009;72:918-927.

19. Aarbiou J, Tjabringa GS, Verhoosel RM, et al. Mechanisms of cell death induced by the neutrophil antimicrobial peptides alpha-defensins and LL-37. Inflamm Res. 2006;55:119-127.
20. Muller CA, Markovic-Lipkovski J, Klatt T, et al. Human alpha-defensins HNPs-1, -2, and -3 in renal cell carcinoma: influences on tumor cell proliferation. Am J Pathol. 2002;160:1311-1324.

21. Gera JF, Lichtenstein A. Human neutrophil peptide defensins induce single strand DNA breaks in target cells. Cell Immunol. 1991;138:108-120.

22. Tani K, Murphy WJ, Chertov O, et al. Defensins act as potent adjuvants that promote cellular and humoral immune responses in mice to a lymphoma idiotype and carrier antigens. Int Immunol. 2000;12:691-700.

23. Holterman DA, Diaz JI, Blackmore PF, et al. Overexpression of alpha-defensin is associated with bladder cancer invasiveness. Urol Oncol. 2006;24:97-108.

24. Balluff B, Rauser S, Meding S, et al. MALDI imaging identifies prognostic seven-protein signature of novel tissue markers in intestinal-type gastric cancer. Am J Pathol. 2011;179:2720-2729.

25. Melle C, Ernst G, Schimmel B, et al. Discovery and identification of alpha-defensins as low abundant, tumor-derived serum markers in colorectal cancer. Gastroenterology. 2005;129:66-73.

26. Gronborg M, Bunkenborg J, Kristiansen TZ, et al. Comprehensive proteomic analysis of human pancreatic juice. J Proteome Res. 2004;3:1042-1055.

27. Schnapp D, Reid CJ, Harris A. Localization of expression of human beta defensin-1 in the pancreas and kidney. J Pathol. 1998;186:99-103.

28. Tiszlavicz Z, Szabolcs A, Takacs T, et al. Polymorphisms of beta defensins are associated with the risk of severe acute pancreatitis. Pancreatology. 2010;10:483-490

29. Tobi M, Kim M, Weinstein DH, et al. Prospective markers for early diagnosis and prognosis of sporadic pancreatic ductal adenocarcinoma. Dig Dis Sci. 2013;58:744-750.

30. Felix K, Fakelman F, Hartmann D, et al. Identification of serum proteins involved in pancreatic cancer cachexia. Life Sci. 2011;88:218-225.

31. Smyth GK. Linear models and empirical bayes methods for assessing differential expression in microarray experiments. Stat Appl Genet Mol Biol. 2004;3:Article 3.

32. Benjamini Y, Hochberg Y. Controlling the false discovery rate: a practical and powerful approach to multiple testing. J R Stat Soc Series B Stat Methodology. 1995;57:289-300.

33. Lundy FT, Orr DF, Gallagher JR, et al. Identification and overexpression of human neutrophil alpha-defensins (human neutrophil peptides 1,2 and 3) in squamous cell carcinomas of the human tongue. Oral Oncol. 2004;40: 139-144.

34. Roesch-Ely M, Nees M, Karsai S, et al. Proteomic analysis reveals successive aberrations in protein expression from healthy mucosa to invasive head and neck cancer. Oncogene. 2007;26:54-64.

35. Jager S, Stange EF, Wehkamp J. Inflammatory bowel disease: an impaired barrier disease. Langenbecks Arch Surg. 2013;398:1-12.

36. Pitchumoni CS, Rubin A, Das K. Pancreatitis in inflammatory bowel diseases. J Clin Gastroenterol. 2010;44:246-253.

37. Okazaki K, Uchida K, Fukui T, et al. Autoimmune pancreatitis - a new evolving pancreatic disease? Langenbecks Arch Surg. 2010;395:989-1000.

38. Zhang Y, Gavriil M, Lucas J, et al. IkappaBalpha kinase inhibitor IKI-1 conferred tumor necrosis factor alpha sensitivity to pancreatic cancer cells and a xenograft tumor model. Cancer Res. 2008;68:9519-9524.

39. Cunliffe RN, Mahida YR. Expression and regulation of antimicrobial peptides in the gastrointestinal tract. J Leukoc Biol. 2004;75:49-58.

40. Raimondi S, Lowenfels AB, Morselli-Labate AM, et al. Pancreatic cancer in chronic pancreatitis; aetiology, incidence, and early detection. Best Pract Res Clin Gastroenterol. 2010;24:349-358.

41. Wang YS, Wang GQ, Wen YJ, et al. Immunity against tumor angiogenesis induced by a fusion vaccine with murine beta-defensin 2 and mFlk-1. Clin Cancer Res. 2007;13:6779-6787.

42. Apte MV, Pirola RC, Wilson JS. Mechanisms of alcoholic pancreatitis. J Gastroenterol Hepatol. 2010;25:1816-1826. 